\title{
|||||||||||||||||||||||||||||||||||||||||||||||||||||||||||||||||.
}

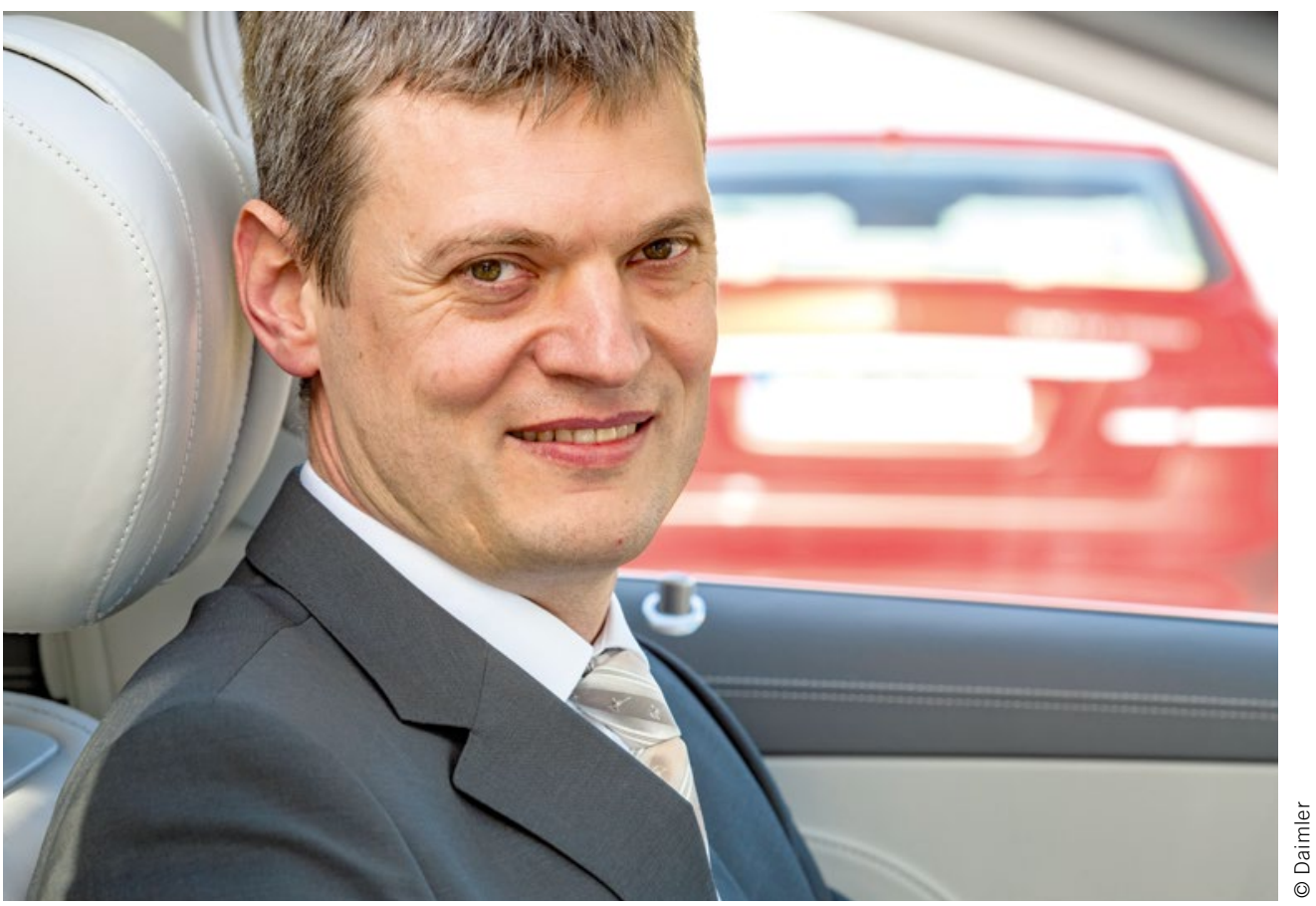

Dr. Michael Hafner

Leiter des Centers Automatisiertes Fahren und Aktive Sicherheit, Mercedes-Benz Pkw-Entwicklung

\section{„Der Wettlauf bleibt spannend“}

Das fahrerlose Taxi wartet bereits um die Ecke und ist im Grunde genommen nur noch eine App entfernt: Diesen Eindruck kann gewinnen, wer den vielfachen Meldungen zum Thema automatisiert fahrende Pkw und Lkw unkritisch folgt.

Gewiss, wir nähern uns diesem Ziel rasant und konsequent. Die automatisierten Fahrfunktionen der Mercedes-Benz S-Klasse, die wir in diesen Tagen in den Markt einführen, wurden praxisgerecht erweitert und sind inzwischen auf fast allen Straßentypen mit nochmals gesteigertem Kundennutzen erlebbar. Vor Kurven, T-Kreuzungen, Kreisverkehren, Mautstellen sowie an Ausfahrten kann das Fahrzeug jetzt die Geschwindigkeit vorausschauend reduzieren.

Möglich wird dies durch das intelligente Einbinden von Kartendaten in die Fahrzeugalgorithmen, durch Verbesserungen bei der Hardware - also beispielsweise Kameras und Radarsensoren mit höherer Performance und größerer Reichweite - und nicht zuletzt durch die immer intensivere Verknüpfung der einzelnen Systeme. Die Basis für den Erfolg liegt natürlich in unserer geballten Erfahrung beim automatisierten Fahren - im Speziellen bei der Softwareprogrammierung der für die Kunden erlebbaren Assistenzfunktionen. Das machen wir seit jeher inhouse und sind daher sehr schnell in der Umsetzung neuer, innovativer Ideen.

Jedoch sind noch einige Hürden zu nehmen, bis ein Fahrzeug ganz ohne Fahrer auskommt. Auch in der neuen S-Klasse ist der Fahrer jederzeit in der Verantwortung. Bis zum fahrerlosen Fah- ren auf einer nicht vorher definierten Strecke und bei allen Verkehrs- und Umweltbedingungen ist es noch ein weiter Weg: Fahrzeugtechnisch, etwa bei der Redundanz von Lenkung und Bremsen, bei den Sensoren, stets aktuellen Kartendaten, bei den Algorithmen und nicht zuletzt auch bei den rechtlichen Rahmenbedingungen. Die Revolution erfordert Evolutionen an vielen einzelnen Stellen.

Wir werden die nötigen Fortschritte rasch und konsequent machen. Unsere Unternehmensstrategie unterstreicht den zentralen Stellenwert des Themas. Wir halten an unserer Vision vom autonomen und unfallfreien Fahren fest.

Eines muss auf dem Weg dahin aber an oberster Stelle stehen - und das ist das Thema Sicherheit. Mit modernen Entwicklungsmethoden wie etwa künstlicher Intelligenz ist es verhältnismäßig einfach geworden, schnell vorführbare Ergebnisse zu erzielen. Der Wettlauf rund um das autonome Fahren wird sich aber erst auf den letzten Metern entscheiden. Dort wird man spätestens Farbe bekennen müssen, ob das System wirklich auch ohne Fahrer unter allen real anzunehmenden Verkehrs- und Umweltsituationen funktional sicher arbeitet.

Aus unseren umfassenden Felderprobungen konnten wir ein sehr realistisches Bild ableiten, womit man im realen Verkehr rechnen muss - und das ist weit mehr, als einem am Schreibtisch einfällt. Ich bin mir nicht sicher, ob ein entsprechend realistisches Bild schon bei allen Playern auf dem Feld des autonomen Fahrens vorherrscht. Der Wettlauf bleibt also spannend! 